\title{
Propuesta de creación de un centro de transferencia de tecnología en la Universidad Politécnica de Gómez Palacio
}

\section{Proposal for the creation of a technology transfer center in UPGOP}

TOVAR-ROSAS, Claudia Rocio †*, GARZA-MOYA, Luis Roberto, ARREOLA-BURCIAGA, Josué Mizraim y DELGADILLO-ALVARADO, Jessica Astridt

\section{Universidad Politécnica de Gómez Palacio}

ID $1^{\mathrm{er}}$ Autor: Claudia Rocio, Tovar-Rosas / ORC ID: 0000-0002-8238-7493, CVU CONACYT ID: 745074

ID $1^{\text {er }}$ Coautor: Luis Roberto, Garza-Moya / ORC ID 0000-0002-5740-1476, CVU CONACYT ID: 68866

ID $2^{\text {do }}$ Coautor: Josué Mizraim, Arreola-Burciaga / CVU CONACYT ID: 769055

ID $3^{\text {er }}$ Coautor: Jessica Astridt, Delgadillo-Alvarado / ORC ID 0000-0003-3197-5188, CVU CONACYT ID: 572720

DOI: $10.35429 / J U M .2019 .10 .3 .35 .44$

Recibido 25 de Octubre, 2019; Aceptado 15 de Diciembre, 2019

\begin{abstract}
Resumen
En la actualidad la mayoría de los estudiantes de las diferentes Instituciones de Educación Superior tienen un compromiso social, el cual es desarrollar nuevas tecnologías que aporten mejoras en la vida diaria de las personas. El objetivo primordial de esta propuesta es crear una interfaz que medié las necesidades de la empresa y las traduzca en áreas de oportunidad y desarrollo para las universidades, e implementar en el aula proyectos dirigidos a las industrias y con ello aportar innovaciones a procesos, productos, entre otros. Actualmente en la Universidad Politécnica de Gómez Palacio, se trabaja con alumnos de cinco diferentes especialidades, los cuales innovan en la creación de nuevos productos, que pueden ser patentados o implementados en la industria. Otro de los rasgos importantes a resaltar es el hecho de que actualmente no existe una forma de proteger autoría de los alumnos, además de que al entregar proyectos terminados por completo, se desconoce el uso que se le dará a dichos productos. Es por está razón que se propone la creación de un centro de transferencia de tecnologías; con él, se estaría promoviendo la innovación de proyectos y participación activa de las empresas, contando con el respaldo de la institución.
\end{abstract}

Innovación, Tecnología, Vinculación

\begin{abstract}
Currently, most of the students of the different Institutions of Higher Education have a social commitment, which is to develop new technologies that contribute improvements in the daily life of the people. The main objective of this proposal is to create an interface that measures the needs of the company and translates them into areas of opportunity and development for universities and to implement projects in the classroom aimed at industries and thereby bring innovations to processes, products, among others. Currently, at the Polytechnic University of Gómez Palacio, students from five different specialties are being worked on, which innovate in the creation of new products, which can be patented or implemented in the industry. Another important feature to highlight is the fact that currently there is no way to protect the authorship of students, in addition to the fact that when delivering completed projects completely, the use that will be given to these products is unknown. It is for this reason that the creation of a technology transfer center is proposed; with it, it would be promoting the innovation of projects and active participation of companies, with the support of the institution.
\end{abstract}

Innovation, Technology, Linkage

Citación: TOVAR-ROSAS, Claudia Rocio, GARZA-MOYA, Luis Roberto, ARREOLA-BURCIAGA, Josué Mizraim y DELGADILLO-ALVARADO, Jessica Astridt. Propuesta de creación de un centro de transferencia de tecnología en la Universidad Politécnica de Gómez Palacio. Revista de Gestión Universitaria. 2019 3-10: 35-44

\footnotetext{
* Correspondencia del Autor (correo electrónico: ctovar@upgop.edu.mx)

$\dagger$ Investigador contribuyendo como primer autor.
} 


\section{Introducción}

En las Instituciones de Educación Superior (IES), se desarrollan proyectos como forma de aplicar los conocimientos, estos están integrados por personas de diversas especialidades, lo que provoca que se enriquezca el conocimiento de los usuarios, tal como indica Galeana (2016), "el Aprendizaje Basado en Proyectos (PBL) implica el formar equipos integrados por personas con perfiles diferentes, áreas disciplinares, profesiones, idiomas y culturas que trabajan juntos para realizar proyectos para solucionar problemas reales.

\section{Estas diferencias ofrecen grandes oportunidades para el aprendizaje y prepararan a los estudiantes para trabajar en un ambiente y en una economía diversos y globales.}

Para que los resultados de trabajo de un equipo de trabajo, bajo el Aprendizaje Basado en Proyectos sean exitosos, se requiere de un diseño instruccional definido, definición de roles y fundamentos de diseño de proyectos.", al aplicar el PBL se aporta al alumno y a los integrantes que participan en este tipo de aprendizaje, las herramientas necesarias para su desarrollo como profesionistas.

Actualmente los alumnos de las diversas instituciones de educación superior producen proyectos en dichas universidades, los cuales en la mayoría de los casos culminan en la realización de un prototipo, pero en ningún momento se respalda la seguridad de su producto, es decir, este puede ser utilizado con propósitos comerciales por algún compañero del mismo entorno e incluso ser tomado por docentes de la institución para su proyección externa a la escuela sin dar el reconocimiento a sus autores, esto ocurre no solamente en instituciones públicas, si no que puede suceder en cualquier centro educativo y de investigación, es por ello que con el Centro de Transferencia Tecnológica (CTT) propuesto se pretende custodiar la propiedad intelectual de dichos proyectos, ya que existe la Ley de Ciencia y Tecnología, la cuál es reglamentaria de la fracción V del artículo 3 de la Constitución Política de los Estados Unidos Mexicanos, ya que salvaguarda todo lo referente a la creación, proceso, implementación y comercialización de los productos generados a partir de una investigación científica.
Está ley comenzó a ser aplicada a partir del 2002, puesto que en dicho año comienza a surgir la necesidad de resguardar a los propietarios del desarrollo de nuevas tecnologías, en cuestión de los tipos de beneficios que podrían obtener a partir de sus investigaciones, así como la diversificación de derechos a partir de la autoría y cooperación, además con ello se promueve la creación de patentes a nivel institucional y local, puesto que existe un amplio desconocimiento sobre las leyes que apoyan a la creación de nuevos productos, además de que actualmente no se cuenta con un centro de trasferencia a nivel local, por lo que la implementación del CTT sería un beneficio para la comunidad estudiantil y científica.

Otro de los fines de la implementación del presente es que a partir de la creación de proyectos, se promueva la unión de las necesidades detectadas en las diferentes empresas tanto locales como nacionales, así como la innovación de soluciones a partir de los desarrollos propuestos en la aulas, los cuales serán enfocados en la resolución de las propuestas, además buscar la inversión necesaria para su procesamiento y con ello se convierta en un producto completo, ya que se estará impulsando la creación de nuevas tecnologías aplicadas a las necesidades detectadas en las diversas comunidades empresariales y científicas.

Estos proyectos al ser desarrollados por estudiantes pueden ser perfectibles a través de la participación de conocedores del área, los cuáles estarán concentrados en el CTT, con esto se logrará crear una cultura de emprendimiento para aumentar el estatus económico de los involucrados, además de aumentar el acervo científico a partir de las nuevas investigaciones y desarrollos de productos.

Además la creación de productos innovadores pueden ser implementados en patentes que apoyen el desarrollo económico e innovador de la región, tal como indica Diessler (2010), "las patentes, históricamente son utilizadas como indicadores para medir el grado de innovación, hoy son consideradas, además, como una valiosa fuente de información científico - tecnológica para la estrategia competitiva". 
Es por ello que además de ser consideradas para el desarrollo profesional son empleadas para aumentar el beneficio económico, tanto para el estudiante, así como para los involucrados en el proceso, tal como lo es la institución escolar y la empresa, ya que al ser comercializados representan una gran inversión para los empresarios y que es redituable en algunos casos a corto plazo.

Todo lo anterior promueve el desarrollo económico de la nación, tal como indica González (2019) "gran parte del trabajo e impulso que se puede dar a los emprendedores surge desde las universidades, ya que son éstas las que tienen más recursos para hacer el proceso de patente que un emprendedor por si solo", en varias Instituciones de Educación Superior (IES) se promueve el crear nuevos productos para ser implementados en la sociedad, con el fin de que los conocimientos no se queden únicamente en el antecedente teórico, si no que se busca el aumentar la práctica a partir de casos de estudio reales, por lo que el desarrollo de un CTT inspiraría a los estudiantes a innovar metodologías y con ello emprender negocios los cuales sean un beneficio para la sociedad en general al generar nuevos empleos y apoyar el comercio nacional.

El presente proyecto representa una importante aportación a nivel escalar, puesto que no se enfoca únicamente en la comunidad universitaria, por lo que representa una gran apoyo al desarrollo regional.

Actualmente se tienen diversas IES las cuales tienen estudiantes en sus diferentes niveles de desarrollo y especialidades, los cuales crean proyectos de investigación, diseño y prototipo; al terminar el ciclo escolar de formación se obtiene tienen un producto comercial - funcional, el cual no tiene la proyección necesaria, puesto que los recursos para su creación en varias ocaciones representa un enorme gasto para sus autores, por ello que la trascendencia de los productos no es la adecuada $\mathrm{y}$ por ende tiene pocos beneficios para sus autores, los mismos que al finalizar su tiempo en la universidad sienten que lo realizado en las aulas es completamente improductivo, además de que no tienen un sustento asegurado para el futuro y esto conlleva a la incertidumbre.
Actualmente se cuenta con algunas empresas como incubadoras de negocio ubicadas en dentro de algunas IES, las cuales tienen un costo excesivo para la creación de empresas, además de que se deben tener algunas características para presentar cualquier tipo de proyecto, ya que estas crecen en mayor forma con el apoyo de recursos gubernamentales, tal como indican Regalado et al. (2016),“ la principal ventaja que actualmente tienen los emprendedores es que pueden disponer de apoyo profesional para llevar a cabo sus ideas de negocio.

Para ello se implementaron modelos como las IE, que ofrecen ayuda a los nuevos empresarios para impulsar la creación de empresas por medio de la generación de un PN que, posteriormente, en caso de requerir apoyos oficiales, debe ser aprobado por la Secretaría de Economía (SE) y entonces recibir el apoyo económico que sirva de base para la creación de una empresa nueva que sea competitiva e innovadora", tal como se indica, la mayoría de las incubadoras obtienen su sustento a partir de convocatorias nacionales para aprovechamiento de recursos gubernamentales y en algunas ocaciones aportaciones de empresas, esto hace que los períodos de tiempo de espera sea en algunas ocasiones un impedimento para la creación de nuevos proyectos y en sí representa un obstáculo enorme en diversas formas, ya que al no contar con suficiente solvencia económica los emprendedores tienden a desechar su idea o en algunos casos ceden sus derechos, hacia usuarios que generalmente explotan de forma productiva la idea y no ofrecen regalías hacia sus creadores.

Los recursos que se pueden asignar por medio de incubadoras de negocios a dichos proyectos son bajos, puesto que los fondos económicos asignados en la mayoría de las empresas a incubar tienden a costar menos de $\$ 100,000.00$, este monto es considerado como base para la mayoría de las convocatorias disponibles a nivel nacional, es por ello que esto representa una suma insuficiente, si es que se cuenta con el costo de la patente, diseño industrial o simplemente por el registro de marca y manufacturado del producto, además de que se debe de considerar el rediseño, comercialización, así como las consideraciones administrativas necesarias para la puesta en marcha de la mercantilización. 
Es por ello que la creación de un CTT representa una opción viable, ya que tal como indican Manjarres et al. (2013), "las relaciones Universidad empresa no solo conllevan a un crecimiento sostenible de los indicadores de competitividad de un territorio sino que también contribuyen con el desarrollo social y económico de las instituciones; en una economía globalizada y altamente competitiva como la actual ha ganado aceptación la idea que la salud económica y social de cualquier sociedad depende de su capacidad de incorporar conocimiento científico y tecnológico. En este contexto, es fundamental la existencia de colaboración e interacciones relevantes entre instituciones productoras de conocimiento científico y tecnológico, tales como Universidades, centros de investigación, y otros agentes, en especial las empresas y las administraciones públicas. De ahí surgen los sistemas de innovación que vincula la ciencia y la tecnológica con el desarrollo socioeconómico, sustentado en la articulación de tres agentes fundamentales: universidades - empresas -gobierno", actualmente el gobierno estatal y federal a optado por impulsar lo que ellos proponen como los cuatro principales ejes, lo cuales están constituidos por:

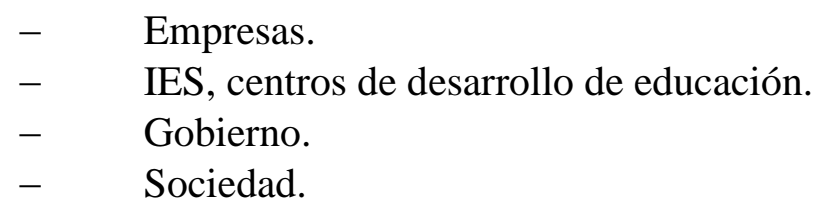

Por lo anterior surge la comunicación de necesidades apremiantes en los diversos ejes, los cuales buscan sean resueltos a partir, de las soluciones que puedan ser aportadas como productos o prototipos desarrollados en las diversas IES y los cuales en conjunto con apoyos económicos puedan solventar las diversas necesidades.

El presente artículo tiene como etapas la introducción al tema en específico, así como el desarrollo de la metodología a seguir y algunos resultados obtenidos por CTT en otros países y regiones, así como los costos de implementación del mismo dentro de la IES y su posible puesta en marcha dentro de los próximos años.

\section{Metodología a desarrollar}

Los pasos realizados para la implementación del presente proyecto fueron los siguientes:
Paso 1: Hacer un estudio de mercado sobre las universidades, empresas, centros de investigación e instituciones gubernamentales a nivel local, los cuáles aporten tanto ideas de proyecto, como recursos económicos para el desarrollo de los mismos.

Según el IMPLAN (2014), actualmente existen en la Comarca Lagunera (Matamoros, Torreón, Gómez Palacio y Lerdo) un total de 31 IES, entre las cuales encontramos instituciones públicas (15) y privadas (16) (Gráfico 1), las cuales imparten clases a los alumnos de Universidad, Posgrados (Maestría y Doctorado) y algunas especialidades, como lo es electrónica, mecatrónica, administración y procesos industriales, para lo cuál se les expide a los participantes como forma de comprobante su título y cédula profesional.

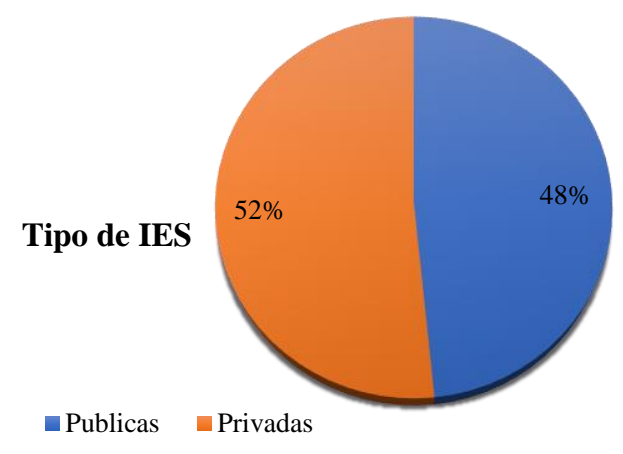

Gráfico 1 Escuelas en la región lagunera

Se tomó en cuenta la región de la Comarca Lagunera, ya que se tienen diversas IES, las cuales ofertan diferentes especialidades y cuyo punto de culminación en algunas de sus asignaturas dependen de la creación de un proyecto o producto final, el cuál genera un prototipo funcional, además de considerar este lugar óptimo, ya que cuenta con varias empresas de diferentes rubros, los cuales buscan apoyar al desarrollo del presente proyecto, puesto que en la el foro de vinculación Durango 2019 promocionado por el COCYTED en Julio, se abarcaron los temas sobre la implementación de soluciones a problemas detectados en las empresas, los cuales fuesen resueltos a partir de proyectos desarrollados en las diversas IES, es por ello que es necesaria la proyección de un CTT, el cual regule la participación de los interesados, además de los niveles de aportación de cada una de las entidades y con ello se promueva el desarrollo científico y económico. 
A nivel nacional se tienen registradas en el Sistema Metropolitano de Indicadores (SIEM) los siguientes datos:

- Grandes empresas con mas de 250 empleados que forman parte de su nómina (Gráfico 2), a nivel nacional el $0.59 \%$ son conformadas por este tipo de empresas, solamente el $1.04 \%$ se encuentra en Coahuila y $0.78 \%$ en Durango.

- Medianas empresas con más de 51 empleados pero menos de 250 , los cuales apoyan a las diferentes áreas dentro de la misma (Gráfico 3), a nivel nacional existen $1.19 \%$ de empresas medianas, además de contar con $1.74 \%$ total en la Laguna.

- Microempresas cuyo máximo de empleados es 50 y con un mínimo de 11 , los cuales realizan diversas actividades industriales menores. (Gráfico 4), a nivel nacional el $92.9 \%$ de empresas que existen son microempresas, en la laguna se tienen $89.65 \%$ de empresas de este rubro.

- $\quad$ Pequeñas empresas, constituidas por un promedio de 0 a 10 personas laborando en dichas empresas.(Gráfico 5), a nivel nacional se tiene registro de que el $5.22 \%$ de las empresas son pequeñas.

Útimos datos de Grandes Empresas en Nacional
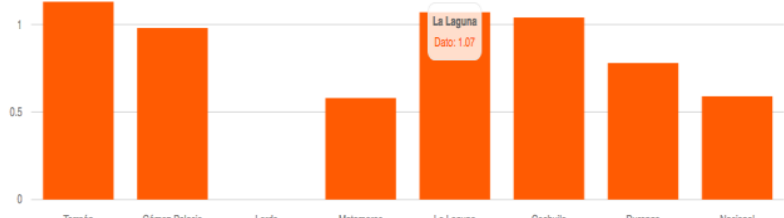

Gráfico 2 Empresas grandes a nivel nacional y local

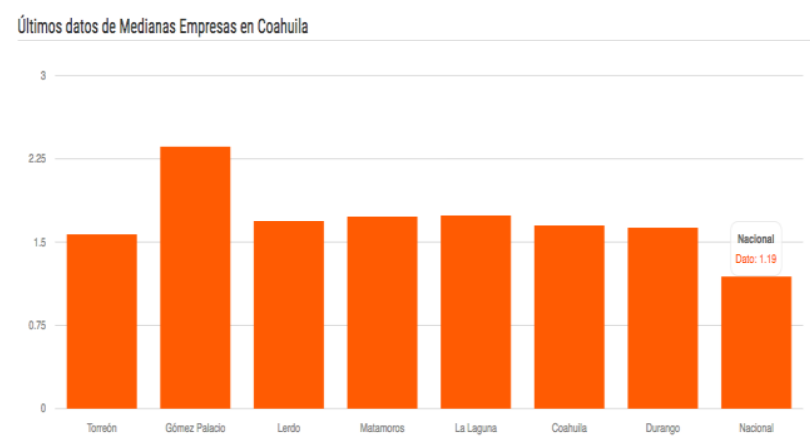

Gráfico 3 Empresas medianas a nivel nacional y local

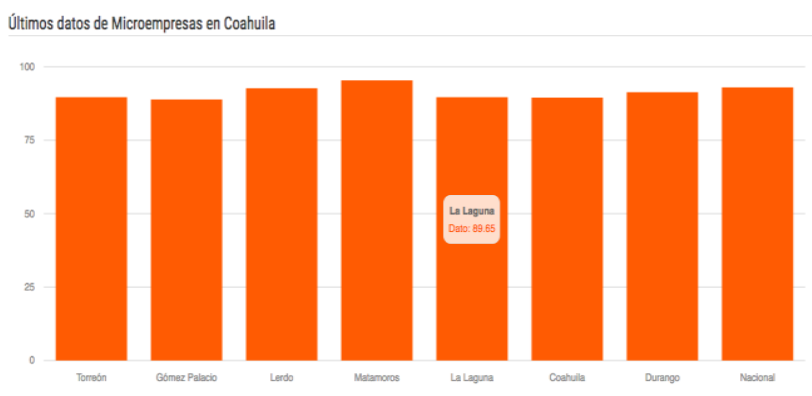

Gráfico 4 Microempresas a nivel nacional y local

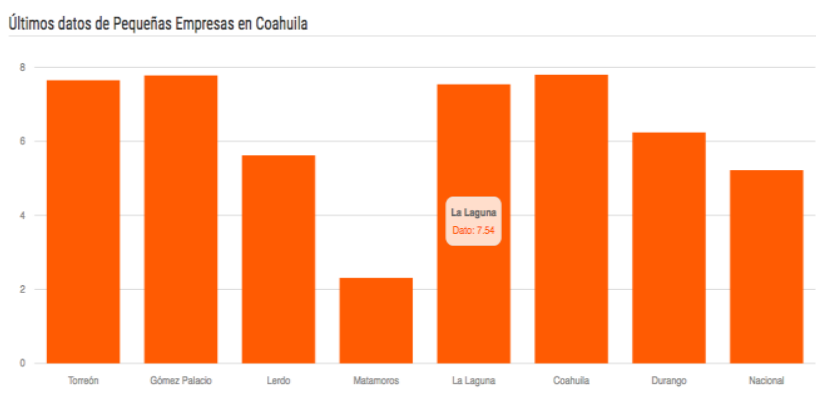

Gráfico 5 Pequeñas empresas a nivel nacional y local

Con el análisis anterior se pudo concluir la efectividad de la implementación de este tipo de CTT, ya que el lugar donde se desarrollará es un área económicamente activa y la cual proveerá no solo de emprendedores, si no de inversionistas que puedan aportar capital económico para el desarrollo de los productos finales, además de que estará sustentado a partir de la La Ley de Ciencia y Tecnología en su artículo 4, fracción XI.

Paso 2: Ubicación del lugar donde se realizará la edificación del CTT, el cual será parte de la Universidad Politécnica de Gómez Palacio (UPGOP), ya que en primer instancia la idea nace en dicha institución y se tienen instalaciones recién construidas, las cuales pueden ser la sede del CTT, además de encontrarse actualmente en un punto estratégico (Figura 1), ya que a pesar de estar en una de las orillas de las ciudades, el paso principal del periférico se encuentra en las puertas de la institución, lo cual aporta una trayectoria para la visita de diversas empresas, además de tener un fuerte lazo con la comunidad lagunera al formar parte activa del IMPLAN y CIESLAG, ambas instituciones sin fines de lucro, la primera apoya actividades que aporten apoyo a comunidades vulnerables y problemas ambientales, entre otros, conformado principalmente por alumnos de diversas IES de la región lagunera, en el segundo caso es una comunidad académica que busca aumentar la investigación y el desarrollo de nuevos modelos educativos e innovaciones. 


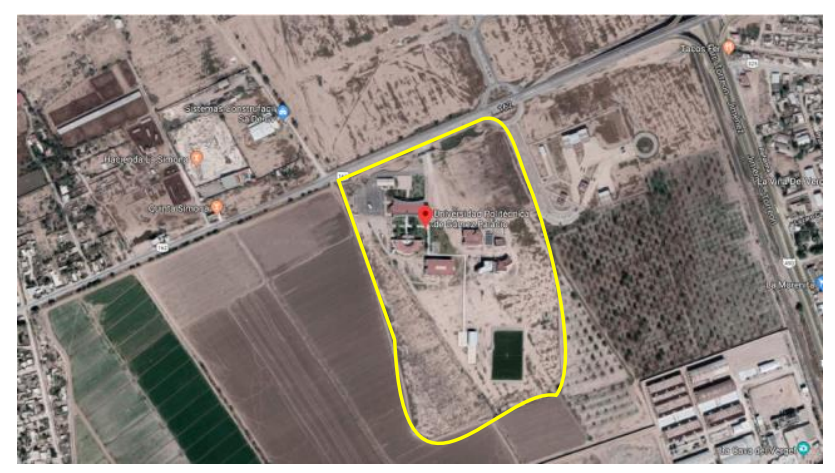

Figura 1 Localización UPGOP

Paso 3: Se analizaron las diferentes empresas que existen a nivel nacional, esto con el fin de organizar las principales unidades económicas que podía apoyar el CTT (Gráfica 6), además de buscar las diferentes especialidades que se ofertan en las IES de la laguna.

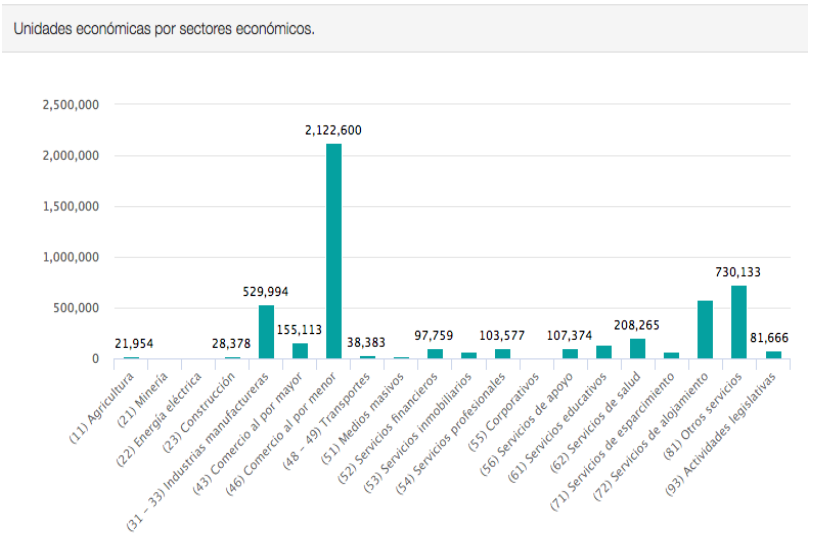

Gráfica 6 Principales sectores empresariales fuente:INEGI ( 2019)

Al tener los datos anteriores se pudo concluir la necesidad de enfocarse en primer instancia a la industria manufacturera, de servicios y en actividades de comercio al por menor, ya que estas son las ramas que tienen una mayor influencia de mercado a nivel nacional.

Paso 4: Se analizó actualmente las políticas a seguir para la realización de dicho centro, para ello se contacto con la Red OTT México (2018), la cual "Es la Red Mexicana de Profesionales en Innovación y Transferencia de Tecnología, una asociación privada sin fines de lucro que agrupa a las Oficinas de Transferencia de Tecnología, así como empresas, organizaciones, profesionistas y emprendedores interesados en apoyar y promover la innovación, comercialización y transferencia de tecnología," esta red tiene objetivos similares a los que se busca realizar al finalizar con el CTT, tales como:

\section{Intercambiar experiencias en el área} transferencia tecnológica.

- $\quad$ Fortalecer el ecosistema de innovación en México.

- Crear una cultura en materia de propiedad intelectual

- Capacitar a los miembros de la Red para su profesionalización y fortalecimiento de competencias.

- Propiciar el desarrollo de nuevas tecnologías y su llegada al mercado.

- Promover la interacción entre la industria, academia, gobierno y sociedad.

Actualmente esta red esta compuesta por varias IES a lo largo de México, además de que cuentan con el apoyo de diversas dependencias de Gobierno, tales como:

Secretaría de economía (SE), la cual apoya en la parte financiera a la creación de proyectos económicamente excesivos, puesto que estos fondos son asignados por el gobierno Federal y tienden a realizar apoyos a emprendedores cuyos proyectos asciendan a montos de más de $\$ 300,000.00$

Consejo Nacional de Ciencia y Tecnología (CONACYT), este organismo público se dedica a impulsar el área de investigación, como principal estrategia tiene el apoyar el desarrollo de proyectos innovadores impulsados por investigadores, docentes y empresarios, esto con el fin de aportar recursos que no sean únicamente económicos, si no que aporten nuevas metodologías a las empresas con el fin de optimizar recursos.

- Instituto Nacional del Emprendedor (INADEM), este instituto existe como apoyo por parte del gobierno para la creación principalmente de PYMES, ya que estos apoyan a los usuarios para realizar planes de negocio, estimaciones de costos e incluso desarrollan actividades de soporte para eficientar las metodologías actuales. 
- Instituto Méxicano de la Propiedad Industrial (IMPI), este organismo cuya principal función es el registro de derechos, presenta una opción recomendable al momento de hacer el registro de marcas y diseños industriales, así como hacer saber sobre los pormenores de las patentes que pueden proceder para su realización como producto.

World Intelectual Property Organization (WIPO), la cual se encarga de el registro de propiedades intelectuales sobre los prototipos y productos que se realicen en las empresas y afuera de ellas.

Paso 5: Actualmente la institución cuenta con mas 300 convenios con diversas IES y empresas, esté es definido y completamente redactado de acuerdo a la especificaciones que se busca tener por parte de los derechos de autor de los alumnos e involucrados en el desarrollo de los proyectos, es decir que en este apartado se definirán los derechos sobre el producto final y que porcentajes se asignaran a cada uno de los actores involucrados.

Paso 6: Se cuenta con políticas que coadyuven a la resolución de problemas y conflictos venideros, ya que solamente con crear el contrato algunas veces se tornará difícil la situación legal de los permisos y de los porcentajes de participación, es por ello que se tomo en cuenta a la hora de buscar el registro de una patente de producto.

Paso7: Se definirán los porcentajes de ingresos, a partir del nivel de participación los cuales deben de ser considerados a partir de la ley de la propiedad industrial, en el cual se establecen los lineamientos para poder realizarlo, además de observar las diversas actividades que pueden generar ingresos, tales como:

$\begin{array}{ll}- & \text { Registro de una marca } \\ - & \text { Dibujo industrial } \\ - & \text { Mejoramiento de un artículo } \\ - & \text { Innovaciones } \\ - & \text { Patentes } \\ - & \text { Nuevos diseños de un producto } \\ & \text { registrado. } \\ - & \text { Entre otros. }\end{array}$

Paso 8: Actualmente se tienen varios colaboradores e inversionistas con interés en la implementación del CTT, estos apoyarán con el diseño del producto, optimización de costos, embotellamiento y traslado, además de considerar la vinculación con otras empresas para la colaboración interna como forma de optimizar tiempos y procesos.

Este paso fue fundamental, ya que se empezó una vinculación con algunas empresas, las cuales al ver los productos creados por alumnos de la institución aportaron capital para su proyección afuera de la institución.

Paso 9: Se concretizó a los investigadores, docentes y estudiantes dentro de las IES, ya que aunque se tienen normas en el reglamento escolar, estos suelen romper las normas y con ello lograr vacíos en las diferentes leyes, además de que en algunas ocasiones copian proyectos y provocan que los creadores principales sean multados en algunos casos por la apropiación de ideas de otros o en algunos ocaciones con la disminución de recursos que podrían haber obtenido si no hubiese ocurrido el plagio.

Lo anterior a ocurrido con frecuencia en algunas IES de la región, es por ello que se tomo como un punto de referencia para la realización del presente y como medida para evitar la famosa "fuga de cerebros" que se tiene actualmente a nivel nacional.

Paso 10: Actualmente se busca un apoyo por parte del gobierno, ya sea federal, estatal o ambos para la puesta en marcha del CTT, actualmente se tienen recursos muy restringidos pero se están llevando a cabo reuniones en donde se argumentan los motivos para subir un poco dichas asignaciones de dinero hacia las IES, es por ello que se debe de formular un escenario en el cual se apoye únicamente con las actuales necesidades para ponerlo en marcha, además de que se han tenido reuniones frecuentes para la inauguración del CTT, pero con negativas por parte del gobierno estatal, ya que no se tienen los recursos económicos disponibles para este tipo de rubro, además de que no se tiene como prioridad el implementar nuevos departamentos en la IES y los permisos necesarios para su funcionamiento, si no que se busca mantener económicamente únicamente el sustento de la universidad. 
Paso 11: Al tener los permisos pertinentes y recursos para la puesta en marcha se buscará una estrategia para la revisión de actividades, así como apoyos económicos, además del personal que dirigirá dicho CTT, los cuales deben de conocer sobre administración de empresas, impuestos, cuestiones legales, entre otros.

\section{Resultados}

Tal como indican Beltrán, Almendarez, Jefferson (2018), "la creación e implementación de políticas públicas orientadas a aumentar el capital intelectual y la innovación podrían beneficiar a una economía emergente como la de México de innumerables maneras", ya que con ello se puede producir una derrama financiera, puesto que al implementar nuevas patentes en el país, estas lograrán aumentar los beneficios económicos recibidos por la nación. Además tal como indican Zulueta, Medina y Negrin (2015), "En la transferencia de tecnologías de la universidad a la empresa se requiere entender las necesidades y oportunidades del conocimiento para ser utilizado por ambos sectores. Es necesario indagar y buscar los conocimientos requeridos con el fin de mejorar el desempeño", ya que con lo anterior directamente se busca el definir un producto a emplear en la industria, que este tenga una aplicabilidad y desarrollo en el tiempo que el alumno sea estudiante, con ello al finalizar su estadía en la institución escolar se obtendrá un producto genuino y que resuelva una problemática en específico, el cual apoyará a la empresa y puede ser motivo para la creación de un negocio establecido por el egresado. Con la implementación de este tipo de proyectos se promueve el amor por la institución que representa y dar el valor que refleja su esfuerzo.

Algunas universidades a lo largo de la república ya han implementado dichos centros, con diversos resultados, además de las diferentes necesidades que se deben contemplar para su implementación, tal como indican García, Aguilera y Catalán (2016) "Los resultados muestran que la transferencia tecnológica en UBB depende de la Capacidad de Investigación de la Universidad - particularmente del número de patentes solicitadas por el investigador principal y el desempeño, por parte del investigador principal, como director de un Centro o Laboratorio de I+D-, además de Redes, en relación al capital social del equipo del proyecto".
La propuesta fue desarrollada en una escuela de educación pública en la cuál se esperaban tener resultados relevantes para la educación y la comunicación de las nuevas tecnologías desarrolladas.

Tal como lo indica la Dra. Salazar (2018), algunas de las interrogantes mayormente abordadas a la hora de hacer un proyecto es si será efectiva la aportación, cual será el impacto, que recursos se llevará, cuanto tiempo será destinado, en el caso de que sea un proyecto que impacte a la población en general cuanto se debe de cobrar por el producto, además de que las instituciones por si solas no puede mantener el coste de una patente, es por ello que son necesarios los centros de transferencia, para poder estar en contacto con empresas inversionistas, con el fin de que puedan ser reproducidos los proyectos y mejorados, además de ser una fuente económica confiable para los egresados y se promueva el uso de patentes, derechos de autor, entre otros.

Aunque algunas instituciones actualmente emplean el uso de centros de transferencia tecnológica, estos llegan a tener altibajos, puesto que dependen de los proyectos a desarrollar por medio de las necesidades detectadas en el entorno, además de los recursos a emplear, tanto por los estudiantes como por los docentes; es por ello que el involucramiento de las empresas es necesario para la continuación de los prototipos en ideas tangibles y certeras.

Actualmente se tiene lista la planeación de las oficinas, el lugar donde se ubicará, así como la propuesta a nivel nacional para el registro, únicamente se está detenido el ejercicio o puesta en marcha del CTT, por motivos de permisos por parte del gobierno federal, ya que este debe otorgar ciertas consignas pertenecientes a la ejecución del mismo, además se espera la pronta resolución por parte de la CGUTyP, ya que uno de sus centros sería modificado para la creación de las instalaciones propuestas.

A lo largo de la creación de esta propuesta se han tenido altibajos, pero se está esperando una resolución que de como resultado la creación del CTT, además de la colaboración con una mayor cantidad de IES y empresas. 


\section{Agradecimiento}

Actualmente el proyecto se encuentra en una etapa inicial, por lo tanto se tiene un agradecimiento especial hacia la UPGOP, IES en la cual se estará desarrollando el proyecto y donde se tiene la edificación para la implementación de dicho CTT, ya que la infraestructura se encuentra construida y lista para el acomodo de los usuarios encargados del centro, además de que se cuenta con la disposición y apoyo, aparte en dicha institución se tiene actualmente un convenio empresarial que puede servir de base para la implementación de la normativa inicial para la creación de los productos finales.

\section{Conclusiones}

Las IES están actualmente teniendo dificultades graves en cuestión de la absorción de ingresos por medio de la creación de proyectos, ya que se han visto reducidos los apoyos gubernamentales, motivo por el cual se deben de comenzar a organizar los proyectos que pueden realizar como equipos estudiantes + docentes +investigadores, esto con el fin de presentarlos a las empresas y con ello se pueda tener un apoyo para el desarrollo de nuevas tecnologías, además de crear nuevos convenios de colaboración con las diferentes empresas no solo a nivel local, si no buscar en toda la nacional si es que existen empresas que puedan apoyar o que detecten necesidades que puedan optimizar con la implementación de dichos proyectos y que sean similares en características.

Además se debe de ver por la seguridad de los alumnos, ya que como se conoce perfectamente, si no se tienen alumnos en las aulas, la razón de ser de las IES desaparece, es por ello que se debe de de proporcionar una medida justa para que los alumnos propongan la creación de nuevas tecnologías, así como se les debe de inculcar dentro de los proyectos integradores la interacciones con otras especialidades, además de inculcar la protección de archivos, firmas electrónicas de los programas o proyectos que realicen, así como el prepararlos para presentaciones de prototipos y la búsqueda de recursos no solo gubernamentales, si no realizar enfoques en donde se tengan que presentar diversos marcos empresariales en los que puedan participar, así como las diferentes vertientes a las que pueden tener acceso como lo es un apoyo industrial, económico, de prototipo, entre otros.
En definitiva, México se encuentra muy detrás en comparación de otros países, ya que actualmente de las patentes que existen a nivel mundial por parte de México, solamente le corresponde al 5\% total Ayala (2017), motivo por el cual se debe de impulsar el derecho sobre la autoría, tanto de inventos que tal vez parezcan simples pero no lo sean, ya que responden a una necesidad en un momento determinado y pueden evolucionar en inventos más grandes.

\section{Bibliografía}

Ayala, F. (28 de Marzo de 2017). Mexico entrega más patentes a EU que a connacionales. Recuperado el 19 de Febrero de 2019, de https://www.publimetro.com.mx/mx/noticias/2 017/03/28/mexicanos-obtienen-5-patentes-eu46.html

Beltrán-Morales, Luis Felipe, AlmendarezHernández, Marco Antonio, \& Jefferson, David J.. (2018). El efecto de la innovación en el desarrollo y crecimiento de México: una aproximación usando las patentes. Problemas del desarrollo, 49(195), 55-76. https://dx.doi.org/10.22201/iiec.20078951e.201 8.195.63191

Diessler, G. (2010). Las patentes como fuente de información para la innovación en entornos competitivos. Información, cultura y sociedad, (22), 43-77.

Galeana, L. (2016). Aprendizaje basado en proyectos. Lourdes Galeana.

González, A. (02 de Enero de 2019). Sugieren explorar modelos para patentes. Reforma, págs. $1-1$.

Implan. (31 de Marzo de 2014). IMPLAN. Recuperado el 13 de Mayo de 2019, de http://www.trcimplan.gob.mx/indicadores-lalaguna/sociedad-universidades.html

INEGI. (01 de Abril de 2019). INEGI. Recuperado el 02 de Julio de 2019, de https://www.inegi.org.mx/temas/directorio/defa ult.html\#Metadatos

Ley de Ciencias y Tecnología. Diario oficial de la Federación. México, 05 de Junio de 2002 
Manjarres, H. L. A., Volpe, B. I. I. \& Altamiranda E. L. A., (2013), Estructura de un centro de transferencia tecnologica: innovacion en una universidad de la costa caribe., Eleventh LACCEI Latin American and Caribbean Conference for Engineering and Technology (LACCEI'2013) International Competition of Student Posters and Paper, August 14 - 16, 2013 Cancun, Mexico.

Pico, J. A. C., Ardila, L. E. B., \& Díaz, P. A. (2009). La transferencia de tecnología en los procesos de investigación de la Universidad Industrial de Santander. Revista GTI, 8(21), 2333.

RedOTT México. (30 de Mayo de 2018). RedOTT. Recuperado el 08 de Julio de 2019, de http://www.redott.mx/\#nosotros

Regalado, E. C., Reynoso, A. M. R., López, M. Z., \& Gómez, P. D. (2016). Las incubadoras de negocios: un modelo económico que ayuda y orienta proyectos empresariales. Revista Iberoamericana de Producción Académica y Gestión Educativa, 3(5).

Salazar Muñoz, Y. (31 de 10 de 2018). Centro de transferencia tecnológica. Desarrollo de proyectos en centros de transferencia de tecnología. (C. R. Tovar Rosas, Entrevistador) Durango, Durango, México.

SIEM. (21 de Octubre de2014). SIEM. Recuperado el 21 de Junio de 2019, de http://www.trcimplan.gob.mx/indicadorescategorias/empresas.html

Zulueta-Cuesta, J. C., Medina-Leon, A., \& Negrin-Sosa, E. (2015). La integración del conocimiento en la transferencia tecnológica universitaria: modelo y procedimiento. Ingeniería Industrial, 36(3), 306-317
TOVAR-ROSAS, Claudia Rocio, GARZA-MOYA, Luis Roberto, ARREOLA-BURCIAGA, Josué Mizraim y DELGADILLOALVARADO, Jessica Astridt. Propuesta de creación de un centro de transferencia de tecnología en la Universidad Politécnica de Gómez Palacio. Revista de Gestión Universitaria. 2019 